\title{
Regenerating new heart with stem cells
}

\author{
Piero Anversa, Jan Kajstura, Marcello Rota, and Annarosa Leri \\ Departments of Anesthesia and Medicine and Division of Cardiovascular Medicine, Brigham and Women's Hospital, \\ Harvard Medical School, Boston, Massachusetts, USA.
}

\begin{abstract}
This article discusses current understanding of myocardial biology, emphasizing the regeneration potential of the adult human heart and the mechanisms involved. In the last decade, a novel conceptual view has emerged. The heart is no longer considered a postmitotic organ, but is viewed as a self-renewing organ characterized by a resident stem cell compartment responsible for tissue homeostasis and cardiac repair following injury. Additionally, HSCs possess the ability to transdifferentiate and acquire the cardiomyocyte, vascular endothelial, and smooth muscle cell lineages. Both cardiac and hematopoietic stem cells may be used therapeutically in an attempt to reverse the devastating consequences of chronic heart failure of ischemic and nonischemic origin.
\end{abstract}

\section{Introduction}

Our understanding of the process of myocardial regeneration is currently under debate. Although the adult human heart is no longer considered a static organ unable to replace its parenchymal cells during the course of life, the rate of myocyte regeneration reported thus far varies dramatically. Minimal levels of myocyte turnover, which decrease with age, have been claimed (1-5), but results have also been obtained supporting continuous myocyte renewal at a remarkable degree (6-12). Independent from the extent of the process, the debate is further intensified by contrasting views regarding the origin of newly formed cardiomyocytes $(13,14)$. These issues have important implications because knowledge of the magnitude of cell regeneration and the mechanisms involved may offer a novel dynamic perspective of cardiac homeostasis and myocardial biology. This information is critical for the identification of strategies aiming at the restoration of the functional and structural integrity of the failing human heart.

The recognition that the adult heart harbors a compartment of multipotent c-kit-positive cardiac stem cells (CSCs) (15-23) and other progenitor cell classes (24-29) capable of differentiating into cardiomyocytes and coronary vessels has raised the challenging question concerning their embryologic origin and role in cardiac cell turnover and regeneration. CSCs are stored in interstitial structures with the characteristics of stem cell niches and can divide symmetrically and asymmetrically, with the ability to self-renew and form a committed progeny $(17,21,30)$. But whether this stem cell pool is actually self-autonomous and fully distinct from HSCs in the bone marrow remains to be determined. c-kit-positive HSCs transdifferentiate and acquire the myocyte, endothelial cell, and smooth muscle cell lineage (31), suggesting that the bone marrow participates in the homeostatic control of the myocardium and the restoration of myocytes and coronary vessels following injury. Additionally, the possibility has been advanced that postmitotic myocytes dedifferentiate, acquire an immature cell phenotype, and then reenter the cell cycle and divide (32-35), representing an alternative or complementary modality of myocyte formation. In this Review, we discuss CSCs, HSCs, and myocyte dedifferentiation as potential mechanisms of myocardial regeneration in the adult organ physiologically and in the presence of pathologic states.

Conflict of interest: The authors have declared that no conflict of interest exists. Citation for this article: J Clin Invest. 2013;123(1):62-70. doi:10.1172/JCI63068.

\section{CSCs}

The shift in paradigm dictated by the discovery of c-kit-positive CSCs has been difficult for the field to accept. The recognition that myocyte regeneration, mediated by activation and lineage specification of endogenous CSCs, is an important determinant of cardiac homeostasis and tissue repair was received with skepticism. Studies suggesting a limited role of myocyte renewal in animals and humans $(2,36)$ were highly publicized with accompanying editorials neglecting CSC function and reiterating the limited nature of myocyte replacement in the adult heart $(37,38)$. The simple concept of a requisite equilibrium between myocyte death and renewal physiologically has often been ignored.

Myocyte apoptosis in the normal human heart involves at least 1 in 100,000 cells (39). Considering that apoptosis lasts at most 4 hours, $0.006 \%$ of myocytes are lost per day, which accounts for a decrease of $2.2 \%$ of myocytes per year. Moreover, myocyte apoptosis increases linearly with age, so that over a period of 30 years approximately $95 \%$ of the original ventricular myocytes are lost as a result of wear and tear of the organ (12). This magnitude of cell death does not include cell necrosis, which has recently been documented independently by the presence of cardiac troponin in the circulation of apparently healthy individuals $(40,41)$. Therefore, a level of myocyte regeneration significantly higher than that predicted by the extent of cell apoptosis is required to preserve cardiac mass and function.

Following the discovery of c-kit-positive CSCs (15), several additional, distinct CSC classes have been described, including ISL1 progenitors, epicardial progenitors, side population progenitors, Sca1 progenitors, progenitors generating cardiospheres, and mesenchymal stem cells $(24-27,29)$. This unusual number of CSC categories is in sharp contrast to the properties of all other self-renewing organs in the organism, in which a single tissue-specific adult stem cell has been found. By definition, stem cells possess well-defined growth properties, suggesting that it is unrealistic that the heart contains such a variety of primitive cells all performing the same biologic function.

Among the different progenitor cell subsets, the c-kit-positive CSC has been well characterized in the mouse (42), rat (15), dog $(16)$, and human $(17,21)$ heart. The expression of the stem cell antigen c-kit is associated with a pool of undifferentiated cells that have essentially identical properties in vitro and in vivo and are indistinguishable among species. Typically, stem cells reside in niches (30), where they are structurally connected to the supporting cells by gap and adherens junctions made by connexins and cadherins, 


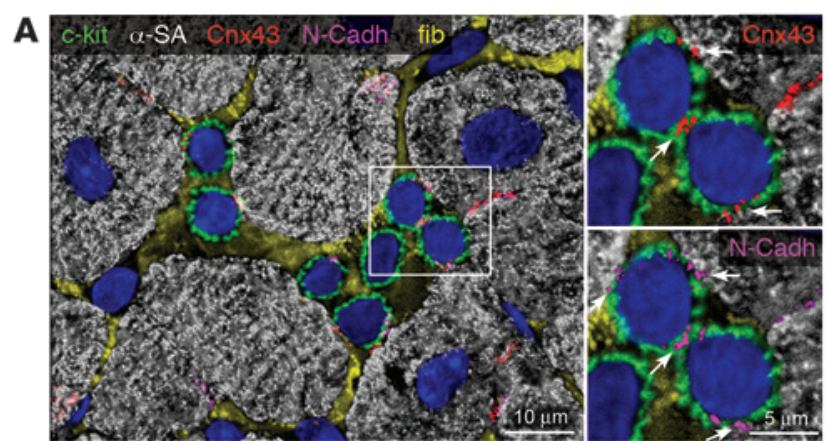

B
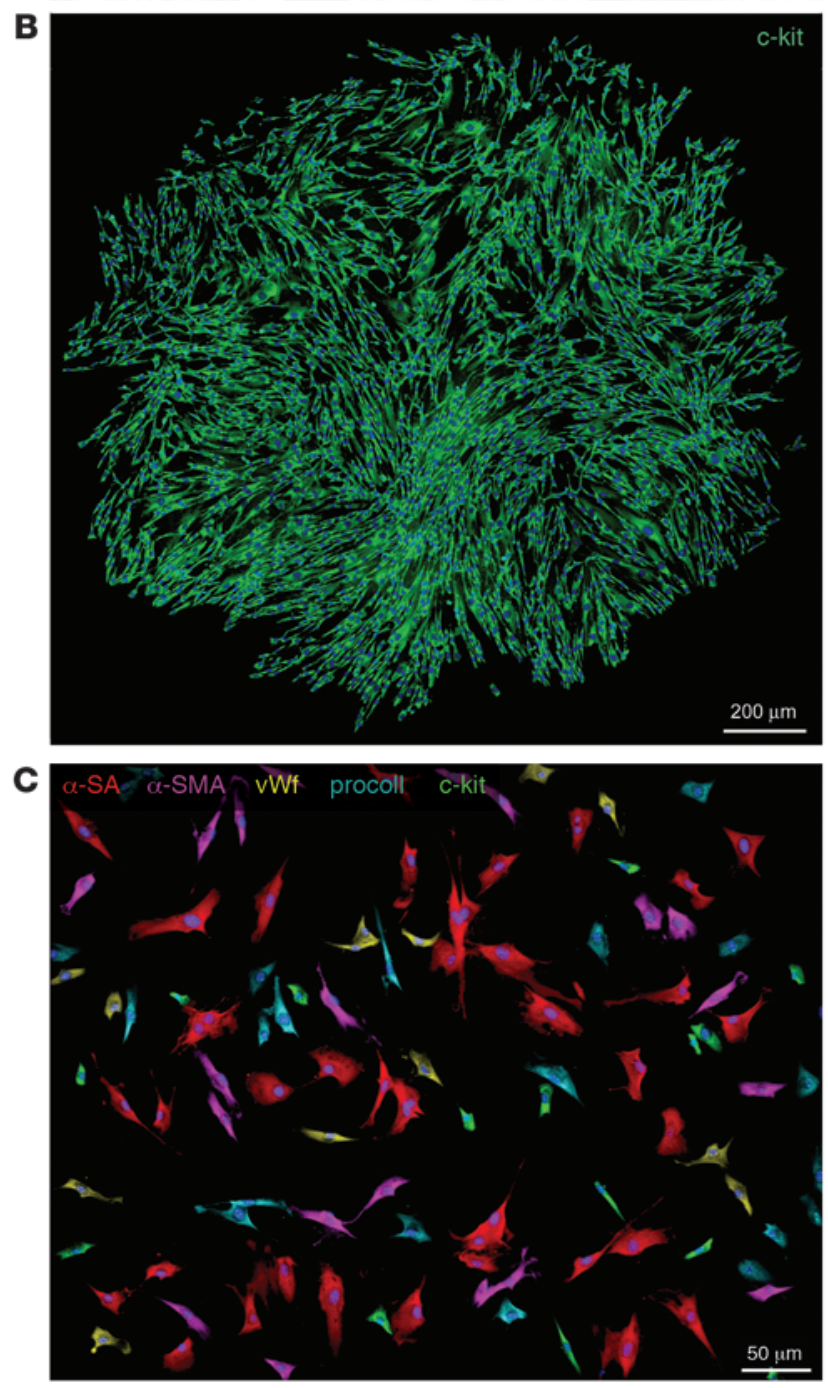

respectively (Figure 1A). The niche constitutes the microenvironment within which stem cells retain their undifferentiated state and receive growth signals from the supporting cells $(43,44)$. Following growth activation, stem cells divide symmetrically or asymmetrically, generating new stem cells and cells destined to acquire specialized functions; c-kit-positive CSCs self-renew, form multicellular clones, and give rise to a differentiated progeny in vitro and in vivo (Figure 1B). By employing viral transduction for clonal tracking of individual mouse and human CSCs, their fate in vivo has been determined (42). The clonality and multipotentiality of

\section{Figure 1}

Human CSCs. (A) Cluster of c-kit-positive CSCs (green) surrounded by fibronectin (fib; yellow). Myocytes are labeled by $\alpha$-sarcomeric actin $(\alpha-S A)$ (white). White rectangle indicates the area shown at higher magnification at right. Scale bar: $5 \mu \mathrm{m}$. Connexin 43 (Cnx43; red) and $\mathrm{N}$-cadherin ( $\mathrm{N}$-Cadh; magenta) are expressed between c-kit-positive CSCs and between CSCs and cardiomyocytes (arrows). Scale bar: $10 \mu \mathrm{m}$. (B) Clone derived from deposition of a single c-kit-positive CSC in a well of a Terasaki plate. Cells in the clone are all c-kit positive (green). Scale bar: $200 \mu \mathrm{m}$. (C) In differentiating medium, clonal c-kitpositive CSCs differentiate into myocytes ( $\alpha$-SA, red), smooth muscle cells ( $\alpha$-smooth muscle actin [ $\alpha$-SMA]; magenta), endothelial cells (von Willebrand factor [vWf], yellow), and fibroblasts (procollagen [procoll], blue). Some undifferentiated c-kit-positive cells (green) are also present. Scale bar: $50 \mu \mathrm{m}$.

c-kit-positive CSCs (Figure 1C) was demonstrated in physiological conditions and following myocardial injury.

This extensive work has provided strong evidence in support of the notion that the adult heart possesses a class of c-kit-positive CSCs that modulates organ homeostasis and conditions tissue repair. During physiological myocardial aging in humans, from 20 to 100 years, the growth and differentiation of c-kit-positive CSCs replaces the entire myocyte compartment approximately 12 times (12). Similarly, activation and myocyte specification of c-kit-positive CSCs occurs in response to myocardial infarction (MI) and chronic aortic stenosis in humans $(7,9,10)$. The crucial role of resident c-kit-positive CSCs in the turnover of cardiomyocytes in the adult rat and mouse heart has also been shown $(30,42,45)$.

The recurrent statement made against the presence of resident CSCs and the significant growth reserve of the human heart is that spontaneous cardiac repair does not occur after infarction and that necrotic tissue is not restored by intact myocardium (14); instead, the healing process results in a thick scar. There is validity in this comment because it describes correctly the progression of $\mathrm{MI}$ and the need for cell therapy with appropriate cells that repair a non-contracting scar with functionally competent cardiomyocytes and coronary vessels. However, a lack of endogenous regeneration after infarction is present in organs including the skin, liver, intestine, kidney, and bone marrow. In all cases, occlusion of a supplying artery leads to scar formation, mimicking cardiac pathology (46-50). In the case of polyarteritis nodosa and vasculitis, microinfarcts develop in the intestine, and skin and resident stem cells do not repair the damaged tissue (51). Infarcts of the bone and bone marrow occur with sickle cell anemia, but HSCs and mesenchymal stromal cells do not reconstitute the structural integrity of the organ $(46,49)$. The simplest example is provided by the occlusion of a branch of a mesenteric artery; a segmental infarct of the intestine develops rapidly, but viable stem cells of the crypts fail to regenerate necrotic tissue (52). The stem cell compartment appears to be properly equipped to regulate tissue homeostasis but does not respond effectively to ischemic injury or, late in life, to aging and senescence of the organ (Figure 2).

Some comments must be made concerning the ISL1-positive cardiac progenitor that has been argued to represent the master human heart stem cell $(27,53)$. The cardiomyocyte specification dictated by the expression of ISL1 defeats the inclusion of ISL1-positive cells into the category of stem cells. As recently stated, adult stem cells are undifferentiated, long-lived cells that have the capacity to self-renew and give rise to a differentiated 
A

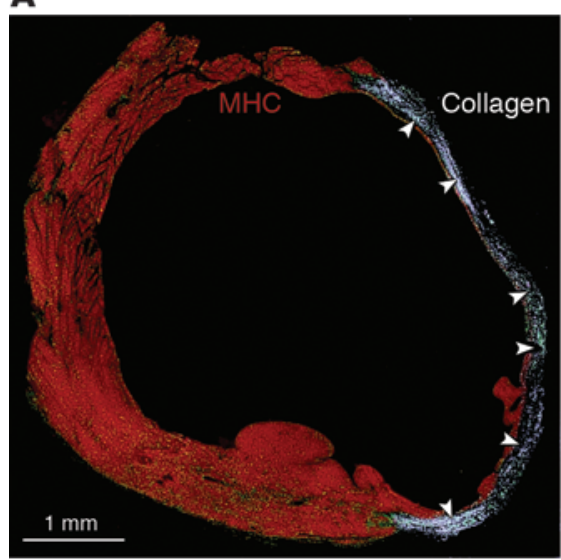

\section{B}
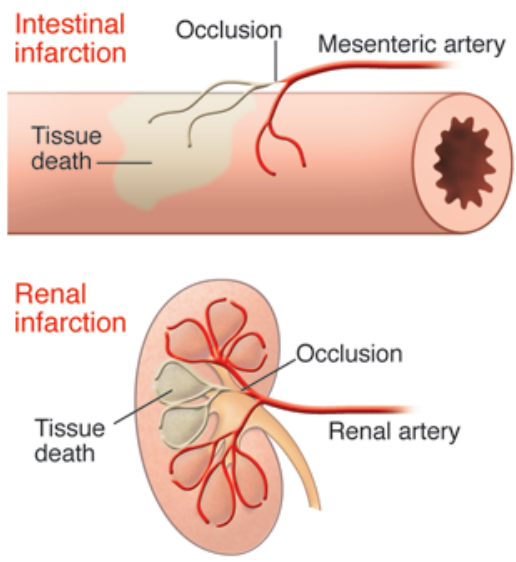

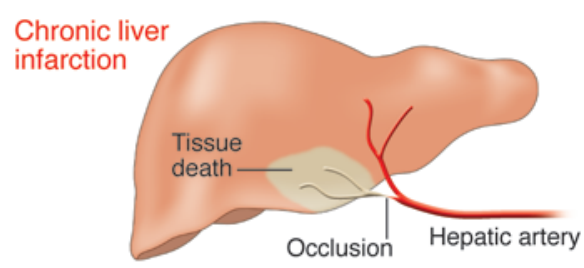

Bone marrow infarction

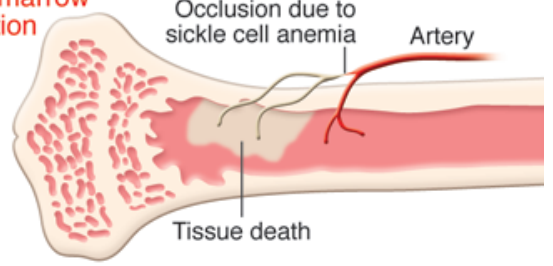

Figure 2

Infarcts in stem cell-regulated organs. (A) Infarcted mouse heart characterized by collagen accumulation (white, arrowheads) in the region of healing. Myocytes are labeled by cardiac myosin heavy chain (MHC, red). Reproduced with permission from Proceedings of the National Academy of Sciences of the United States of America (ref. 72; copyright 2001, National Academy of Sciences, USA). (B) Schematic representation of infarcted tissue in various organs. Regeneration of the infarct is absent in all cases.

progeny (54). ISL1-positive cells are not clonogenic, forming at best small, abortive colonies in vitro, and their functional importance in vivo remains to be shown. ISL1-positive cells are restricted to the embryonic fetal heart and are not present after birth (55), raising questions about their relevance to the treatment of heart disease in children and adults. Similar limitations apply to the other categories of cardiac progenitors; whether they regulate cardiac cell turnover physiologically and promote tissue repair following myocardial injury has not been documented as of yet. At present, the c-kit-positive CSC is the only candidate that fulfils the criteria of tissue-specific adult stem cell $(54,56)$.

Lineage tracing has been viewed as the gold standard for recognizing the properties of stem cells (57). With this approach, the expression of a fluorescent reporter gene is placed under the control of promoters coding for myocyte and vascular proteins to track the origin of cells and their destiny in animals in which genetic manipulations can be routinely performed (Figure 3). Unfortunately, this protocol cannot document whether stem cells divide asymmetrically, i.e., self-renew, or whether the labeled progeny derive from activation of a single or several stem cells, i.e., unipotency or multipotency $(13,42,58,59)$. Additionally, results from fate-mapping strategies cannot be taken at face value. Fate mapping indicates that no myocyte renewal in the mouse heart occurs up to 1 year of age (36), despite continuous myocyte death by apoptosis and necrosis $(45,60)$. This conclusion is in stark contrast to pulse-chase labeling studies with thymidine analogs and mathematical modeling of hierarchically structured cell populations, both of which document constant formation of new myocytes $(12,30,42,45)$. Most importantly, lineage-tracing protocols cannot be implemented in humans.

\section{HSCs}

The recognition that CSCs reside in the adult myocardium poses the question of their origin and mechanisms of growth activation. CSCs may be present early during development and be responsible for cardiomyogenesis in embryonic and fetal life, a role that may continue postnatally and into adulthood. Conversely, these undif- ferentiated cells may be located in distant organs, in particular the bone marrow, and continuously translocate to the developing and mature heart, where they assume specific functions, leading to the formation of the mature cardiac phenotype during development and to the preservation of tissue homeostasis in the adult organ.

Recent results favor the notion that cardiac development is controlled mainly by growth and differentiation of c-kit-positive CSCs, which are largely responsible for the formation of the myocyte progeny present at birth (61). Spontaneous $\mathrm{Ca}^{2+}$ oscillatory events that occur within CSCs promote cell cycle reentry and asymmetric stem cell division $(61,62)$. HSCs appear not to be an important determinant of cardiomyogenesis, but the possibility that the bone marrow contributes partly to the maturation of the embryonic, fetal, and neonatal heart cannot be excluded. Similarly, bone marrow-derived cells are involved in the adaptive response of the adult heart following ischemic and nonischemic myocardial injury (63, 64), although the magnitude of the process is currently unknown.

The possibility that HSCs are involved in the restoration of damaged myocardium and contribute to the cardiac repair process suggests that bone marrow-derived cells are capable of forming cardiomyocytes and coronary vessels in spite of their predetermined lineage specification. During prenatal life, stem cells undergo a hierarchical progressive restriction of developmental options, and this mechanism of embryonic specification was thought to be irreversible and inviolable in adulthood $(65,66)$. However, this notion has been challenged by several examples of transition from one cell type to another or, more unexpectedly, from one cell lineage to another lineage (67-70). The unanticipated plasticity of adult HSCs to generate cells beyond their own tissue boundary became the driving force of a series of studies in which HSCs were implemented experimentally to restore the necrotic myocardium after infarction (71-78).

The notion that HSCs transdifferentiate and form cardiomyocytes and coronary vessels has been sharply attacked, and criticisms have been made, regarding the implementation of this form of cell therapy in human beings (79-82). However, based on positive results obtained in phase 1 and phase 2 clinical trials $(83,84)$, 
A
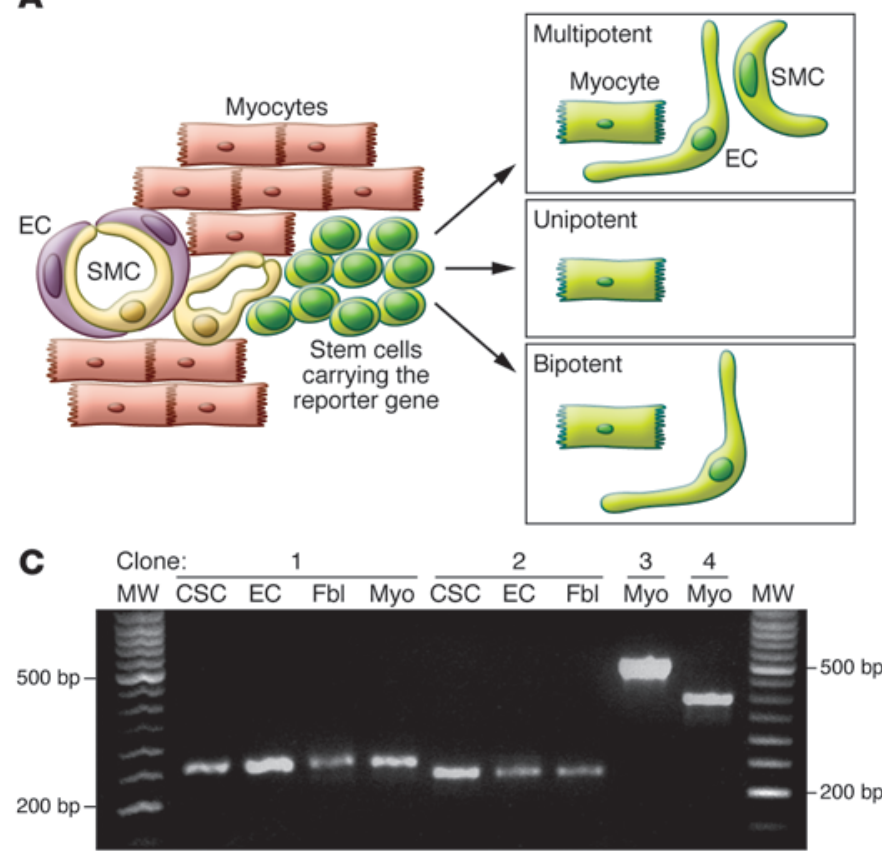

B
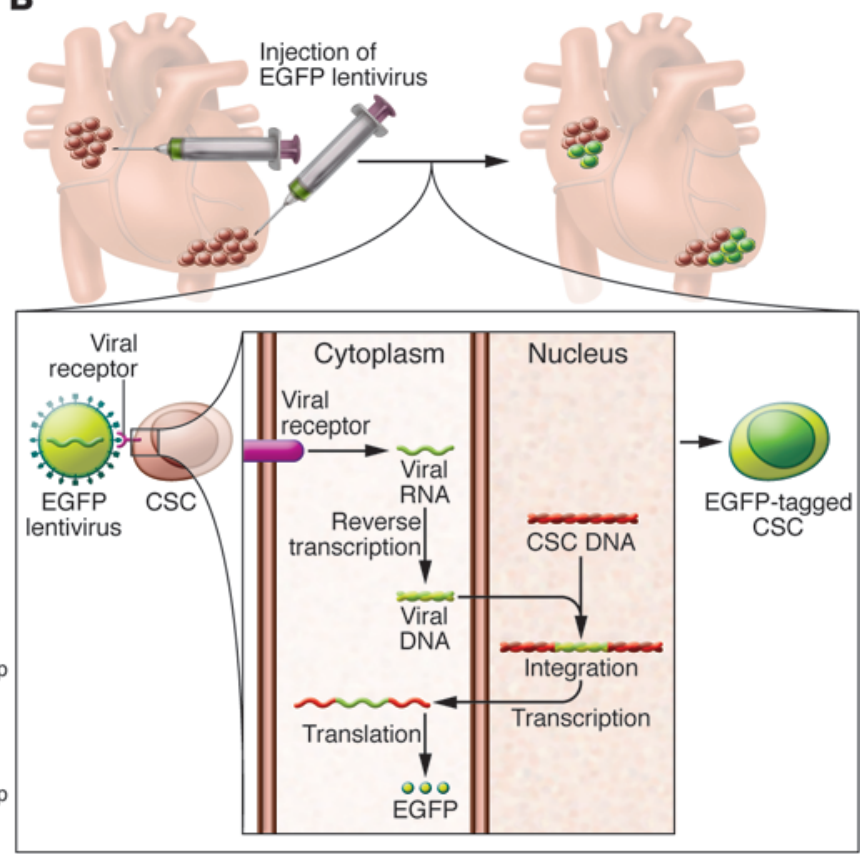

Figure 3

Lineage tracing of CSCs. (A) Representation of fate mapping involving the use of a fluorescent reporter gene (EGFP, green) driven by an inducible promoter coding for a stem cell-specific protein. Following differentiation and loss of stem cell antigen, myocytes, ECs, and SMCs continue to express EGFP, indicating a lineage relationship between ancestors and descendants. However, the labeled progeny may derive from activation of one or several stem cells, failing to document the multipotency of the parental stem cell. (B) Representation of viral gene tagging. Infection of CSCs with EGFP lentivirus results in the semirandom insertion of the proviral integrant in the genome of the recipient cell. Transcription and translation of the viral DNA result in expression of EGFP and fluorescent labeling of the infected CSCs. The unique insertion site of the viral genome is inherited by the entire population derived from the parental cell and can be amplified by PCR. CSCs nested in atrial and apical niches were labeled in situ to identify their progeny in vivo. (C) Four distinct clones were identified in EGFP-tagged CSCs, ECs, fibroblasts (Fbl), and cardiomyocytes (Myo) isolated from the ventricle of one mouse heart. Multiple PCR products (bands in agarose gel) were identified. Bands of the same molecular weight correspond to identical sites of integration of the proviral sequence in the host genome of CSCs, myocytes, ECs and fibroblasts, documenting the multipotency of CSCs in vivo. Reproduced with permission from Proceedings of the National Academy of Sciences of the United States of America (ref. 42; copyright 2009, National Academy of Sciences, USA).

different classes of bone marrow cells are currently being used in patients with acute and chronic ischemic cardiomyopathy. A phase 3 trial supported by the European Union is ongoing (BAMI; http://www.euram.ltd.uk/BAMI/). But whether the apparent benefit of bone marrow-derived cells is dictated by the ability of these cells to regenerate damaged myocardium is the subject of intense debate. The predominant view is that these cells secrete a variety of cytokines that activate endogenous progenitors, which are actually responsible for the repair process and the improvement in ventricular function (82). Unfortunately, the documentation of the paracrine effects mediated by bone marrow cells in animals has been elusive at best, and data in humans have not helped to resolve this conundrum. Findings have been reported that support the notion that HSCs acquire the myocyte lineage and differentiate into functionally competent cardiomyocytes $(75,85)$, strengthening the view that HSCs may have therapeutic import for the human disease by acquiring the myocyte and endothelial cell lineage (Figure 4). Despite animal and human results, myocardial repair continues to be viewed with suspicion and trepidation. The traditional view of the ability of the heart for proliferative growth was defined as "on shadings between none and almost none" (86), whether it derives from activation of resident CSCs or HSCs.

\section{Myocyte dedifferentiation}

Myocytes with decreased myofibrils and expansion of the undifferentiated cytoplasm have been found in pathological cardiac hypertrophy (9), idiopathic dilated cardiomyopathy (IDC), and acute MI $(7,35)$ and in the presence of hibernating myocardium (87). Partial loss in the normal distribution pattern of titin, desmin, and cardiotin has been reported, together with the reexpression of fetal genes, including $\alpha$-smooth muscle actin, atrial natriuretic peptide, $\alpha$-skeletal actin, and $\alpha$-smooth muscle actin $(35,87)$. Cardiomyocytes with these structural characteristics have been interpreted as the product of cell dedifferentiation, resulting in the acquisition of an immature proliferative cell phenotype. Dedifferentiation of adult cardiomyocytes has also been claimed following inhibition of p38 MAPK (32), myocardial injection of the extracellular matrix component periostin (33), or the growth factor neuregulin (34), although the effect of periostin on myocyte replication in vivo has been challenged (88).

Myocytes essentially identical to those commonly involved in the prenatal and early postnatal development of the myocardium (89, 90) reappear in the diseased human heart, and this process may be mediated by the release from macrophages of a member of the IL- 6 inflammatory cytokines, oncostatin M (35). Surprisingly, divid- 


\section{A}

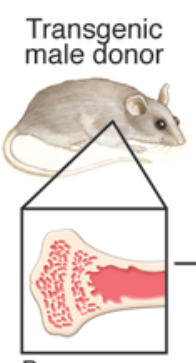

Bone marrow

B
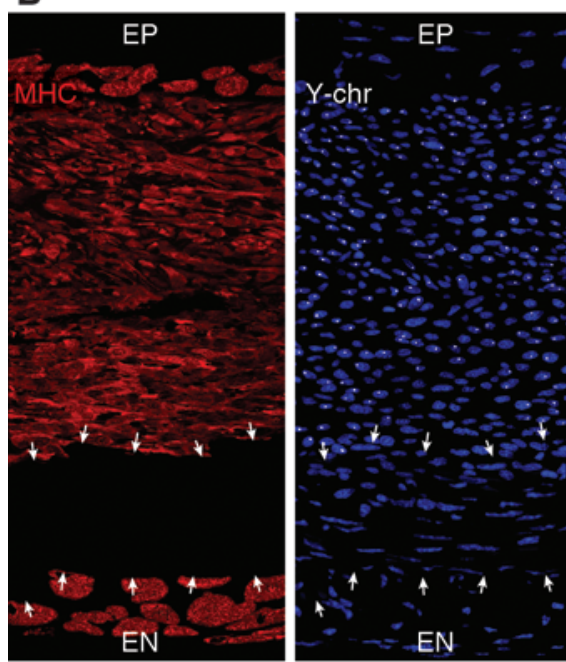

C
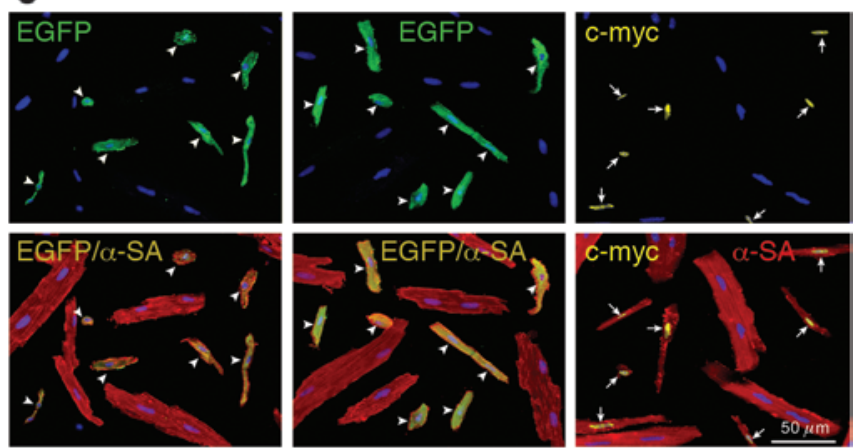

D

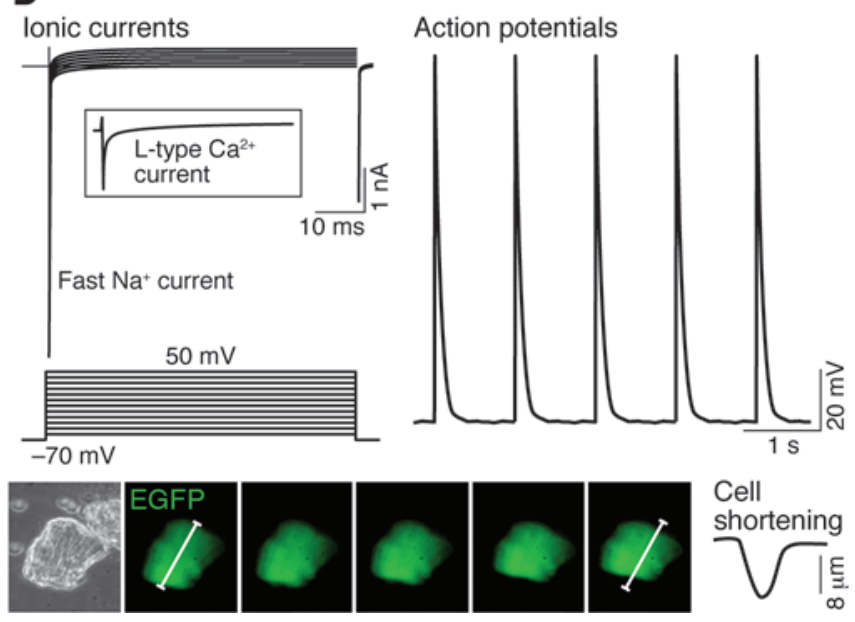

\section{Figure 4}

Transdifferentiation of c-kit-positive HSCs. (A) Schematic representation of transgene constructs used in the generation of donor mice for the acquisition of HSCs to be delivered after infarction. In each case, the promoter that controls the ubiquitous ( $\beta$-actin) or myocyte-restricted $(\alpha-\mathrm{MHC})$ expression of the transgene (EGFP or c-myc-tagged nuclear-targeted Akt) is shown. c-kit-positive HSCs from donor males were injected intramyocardially in wild-type female infarcted mice. NLS, nuclear localization signal (ref. 75). (B) Infarcted female mouse treated with male HSCs. Regenerated myocytes (left; MHC, red) carry the $\mathrm{Y}$ chromosome (center; Y-chr; white dots in nuclei). Merged image is shown at right. Arrows indicate non-regenerated infarct. A thin layer of spared myocytes is present in the epimyocardium (EP) and endomyocardium (EN) (ref. 75). Scale bar: $50 \mu \mathrm{m}$. (C) Examples of myocytes isolated from the regenerated myocardium of mice injected with HSCs collected from $\beta$-actin EGFP, $\alpha$-MHC EGFP, or $\alpha$-MHC c-myc-tagged nuclear-targeted Akt mice. Top panels illustrate the localization of EGFP (left and middle, green; arrowheads) and c-myc (right, yellow nuclei; arrows) in newly formed cardiomyocytes. Bottom panels show the colocalization of $\alpha$-SA and EGFP (yellow, arrowheads) and of $\alpha$-SA and c-myc (yellow nuclei, arrows). Large spared myocytes negative for EGFP or c-myc are also present (ref. 75). Scale bar: $50 \mu \mathrm{m}$. (D) Myocyte-specific ionic currents and action potentials of a cell isolated from the regenerated infarcted myocardium. EGFP-positive myocytes show contractile activity (bottom). Original magnification, $\times 200$. Reproduced with permission from Proceedings of the National Academy of Sciences of the United States of America (ref. 75; copyright 2007, National Academy of Sciences, USA).

ing myocytes repeatedly observed in IDC and acute and chronic MI in humans $(6,7,10)$ have not been detected (35). Additionally, enhanced delivery of oncostatin $\mathrm{M}$ by macrophages in an in vivo model of dilated cardiomyopathy or following MI recapitulates the fetal-neonatal cell program but fails to promote myocyte regeneration (35), questioning the mechanisms involved in the negative (dilated myopathy) and positive (MI) effects of myocyte dedifferentiation on ventricular remodeling and cardiac hemodynamics. Similarly, the dedifferentiated cardiomyocytes identified in the human heart with IDC or acute MI never reached karyokinesis or cytokinesis. The expression of the fetal gene program with hemodynamic overload may simply reflect regenerated myocytes derived from commitment of resident CSCs rather than dedifferentiation of terminally differentiated postmitotic myocytes, which acquire an unwanted, mechanically inefficient cell phenotype (13).

Oncostatin $\mathrm{M}$ has also been argued to reverse in vitro the structural organization of postmitotic myocytes, resulting in the upregulation of several stem-related genes, including c-kit (35). Although the c-kit protein was never identified and the potential contamination of culture preparations from resident c-kit-positive progenitor cells was not excluded, a reinterpretation was immediately offered for the data on c-kit-positive CSCs (91). In the absence of in vivo findings, and despite the disclaimer from the authors, the validity of multiple demonstrations of resident c-kit-positive CSCs (15-23) was challenged (91). The rationale for this scientific view is obscure at best.

Recently we tested whether fetal myocytes, obtained from mice at E16-E18 expressing EGFP under the control of the c-kit promoter, were able to dedifferentiate and reacquire a degree of stemness, including the expression of c-kit (61). A total of 102 myocytes in mitosis were examined and none showed EGFP labeling. Moreover, non-dividing fetal myocytes expressing different levels 
A
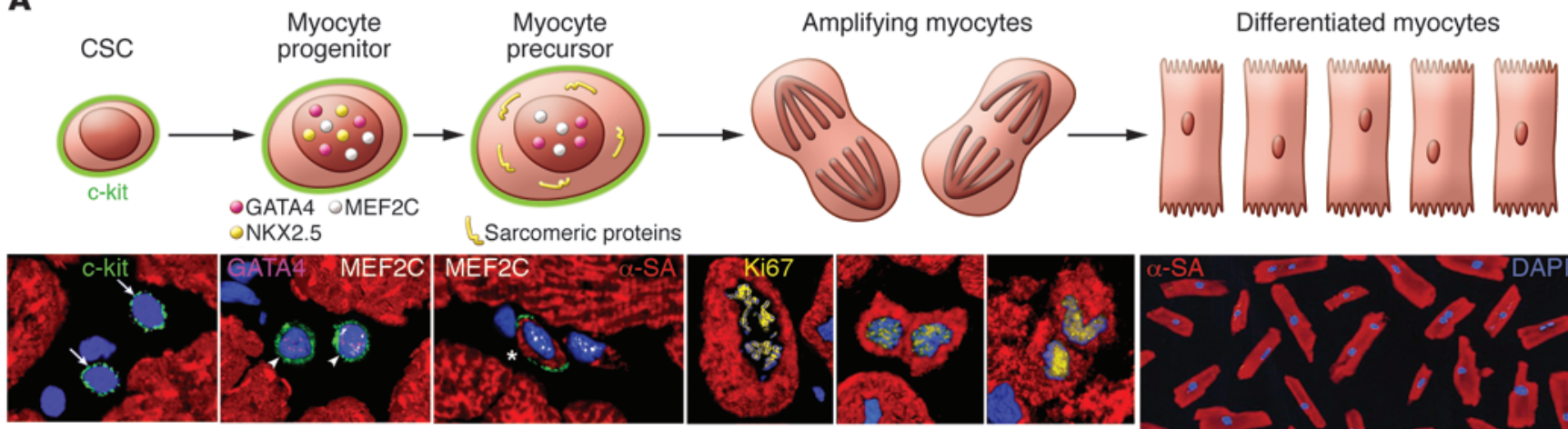

B
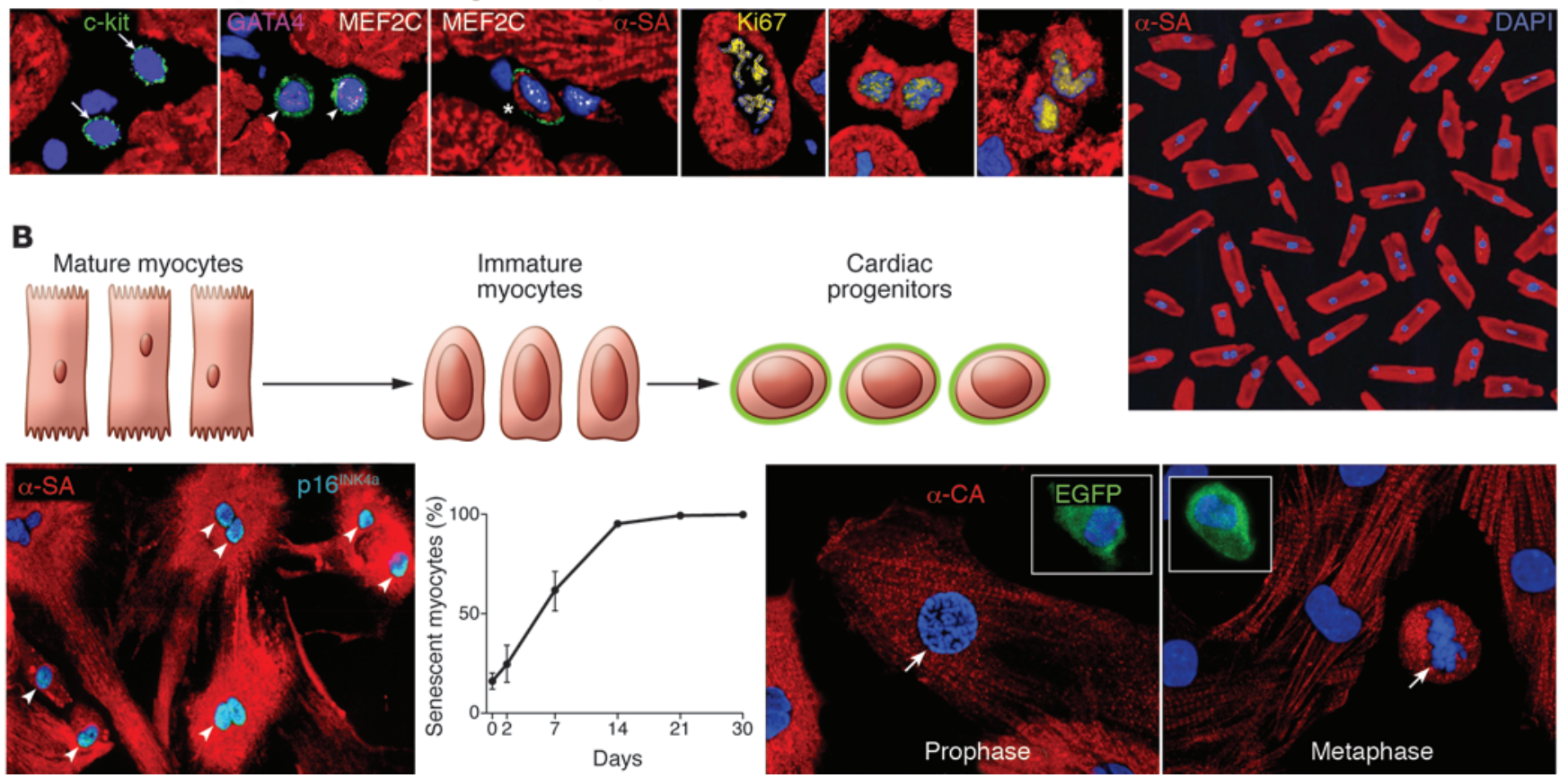

Figure 5

Cellular mechanisms of myocardial homeostasis. (A) Lineage relationship between CSCs and cardiomyocytes: CSC commitment results progressively in the formation of myocyte progenitors, myocyte precursors, and transit-amplifying myocytes, which divide and differentiate into mature myocytes. CSCs are c-kit-positive lineage-negative cells. Progenitors express c-kit and myocyte-specific transcription factors (GATA4, Nkx2.5, MEF2C) but do not show sarcomeric proteins. Precursors possess c-kit, myocyte-specific transcription factors, and sarcomeric proteins. Amplifying cells have myocyte nuclear and cytoplasmic proteins but are c-kit negative. This hierarchy is shown by immunolabeling and confocal microscopy (bottom). Micrographs from left to right show lineage-negative CSCs (arrows); myocyte progenitors (arrowheads) expressing c-kit, GATA4, and MEF2C; myocyte precursor (asterisk) showing c-kit, MEF2C, and $\alpha-S A$; and three transit-amplifying myocytes ( $\alpha$-SA). Chromosomes are organized in anaphase-telophase and are Ki67 positive. Original magnification, $\times 700$. Terminally differentiated cardiomyocytes from the human heart are shown at far right. Original magnification, $\times 100$. Reproduced with permission from Proceedings of the National Academy of Sciences of the United States of America (ref. 9; copyright 2003, National Academy of Sciences, USA). (B) The postulated process of dedifferentiation of postmitotic myocytes to immature myocytes and cardiac progenitors is represented schematically. Micrographs document that culture of differentiated myocytes is not coupled with reentry into the cell cycle and reversal to a primitive state. From left to right, adult cardiomyocytes in vitro show disassembly of the contractile apparatus and express the senescence-associated protein p16 INK4a (bright blue, arrowheads; original magnification, $\times 250$ ). The percentage of senescent myocytes increases with time. Fetal myocytes, isolated from a reporter mouse in which EGFP is driven by the c-kit promoter, divide in vitro (arrows) but do not express c-kit. Original magnification, $\times 500$. Insets show c-kit-positive EGFP-positive CSCs used as a positive control. Reproduced with permission from Circulation Research (ref. 61).

of sarcomeric proteins were all EGFP negative, excluding cell dedifferentiation. Importantly, the generation of myocytes by dedifferentiation of mature cells, if it occurs, would have a minor role in physiological cell renewal, being restricted to cardiomyocytes (Figure 5). Similarly, dedifferentiated myocytes would be severely limited in their ability to sustain or improve ventricular performance following injury. Myocytes in the absence of adequate vascular supply and tissue oxygenation would be mechanically inefficient. Conversely, CSCs are multipotent and form in a coordinated manner cardiomyocytes and coronary vessels, fundamental components of myocardial repair.

\section{Future directions}

Endothelial progenitor cells, mononuclear bone marrow cells, mesenchymal stromal cells, and CD34-positive cells have been administered to patients suffering from acute MI or chronic ischemic cardiomyopathy $(83,84)$. Based on meta-regression analyses, these interventions have had positive outcomes, documenting the feasibility and safety of this therapeutic strategy and, in most cases, the beneficial effects on cardiac function. An average significant increase in ejection fraction of 3 percentage points has been reported $(83,84)$, although studies have also been published that indicate no changes in ejection fraction (92-96). Although enroll- 
ment is ongoing for a large clinical trial to test the effect on mortality of the delivery of mononuclear bone marrow cells (BAMI; http:// www.euram.ltd.uk/BAMI/), the documentation of tissue-specific adult CSCs offers an alternative and theoretically more effective form of cell therapy for patients with chronic heart failure (CHF). Based on this premise, a phase 1 clinical trial with autologous CSCs is in progress (ClinicalTrials.gov NCT00474461), and the preliminary results are encouraging (97). Additionally, difficulties do not appear to exist in the acquisition of small myocardial samples in humans or in the isolation and expansion of CSCs in quantities that can be employed therapeutically and repeatedly over time (98).

However, multiple variables may negatively affect the growth reserve of resident CSCs in the aging, decompensated heart, limiting their regenerative potential (99-101). The amplification in vitro of c-kit-positive human CSCs yields a cell population composed of stem cell subsets with considerably different ability to divide and differentiate. The association of c-kit with distinct membrane receptors conditions the phenotypic plasticity of CSCs and their ability to form mechanically efficient cardiomyocytes (101). Aging is associated with a progressive decrease in the compartment of human CSCs with high regenerative potential, and with a progressive increase in the pool of stem cells with minimal or no ability to divide (12, 99-101). The loss of CSC function with aging is mediated partly by an imbalance between factors promoting growth and survival and factors enhancing oxidative stress, telomere attrition, and apoptosis. However, a small pool of CSCs with significant growth reserve persists in the senescent and decompensated heart $(12,98,102)$. Two growth factor receptor systems have a critical role in CSC replication, differentiation, senescence and death: the IGF1/IGF1 receptor system and the renin-angiotensin system (RAS). The IGF1/IGF1 receptor induces CSC division, upregulates telomerase activity, maintains telomere length, hinders replicative senescence, and preserves the population of functionally competent CSCs in animals (45) and humans (101). The expression of IGF1 receptors and the synthesis of IGF1 are attenuated in aging CSCs, possibly diminishing the ability of IGF1 to activate cell proliferation and interfere with oxidative damage and telomeric shortening (60).

The documentation that a local RAS is present in human CSCs and that formation of Ang II and expression of Ang II receptors increase with age provides evidence in favor of the role that this octapeptide has in CSC senescence and death. Ang II generates reactive oxygen species, resulting in DNA damage; 8-OH-deoxyguanosine (8-OH-dG) accumulates at the GGG triplets of telomeres, resulting in telomeric shortening and uncapping (103). Conversely, IGF1 decreases oxidative stress (60) and repairs DNA damage by homologous recombination (104). Therefore, changes in the proportion of these growth factor receptor systems in CSCs influence their growth reserve, a critical determinant of stem cell-based therapy.

Importantly, CSCs cannot be implemented in acute events in view of the time required for their preparation, whereas mononuclear bone marrow cells constitute an appealing form of cell intervention. Mononuclear bone marrow cells can be easily collected from bone marrow aspirates or the peripheral blood upon their mobilization from the bone marrow with cytokines. However, mononuclear bone marrow cells should not be confused with c-kit-positive HSCs that constitute a minute fraction of the mononuclear bone marrow cell pool (105). Experimentally, remarkable levels of myocardial regeneration after infarction have been obtained with c-kit-positive $\operatorname{HSCs}(71,72,75,85)$ but not with mononuclear bone marrow cells (106). At present, it is unknown whether CSCs and HSCs are similarly effective in reconstituting the necrotic and scarred tissue, or whether limitations exist in CSC growth and HSC transdifferentiation, resulting in inadequate restoration of the damaged myocardium. A fundamental question to be answered is whether CSCs are superior, equal, or inferior to HSCs for the regeneration of myocytes and coronary vessels in animal models of CHF.

The therapeutic efficacy of these two stem cell classes depends on their ability to survive in the hostile milieu of the damaged heart, to engraft within the myocardium, and to grow and differentiate. HSCs may have a growth potential that is superior to CSCs, but transdifferentiation could affect this characteristic, and CSCs may constitute a more powerful form of therapy for cardiac repair. The process of transdifferentiation may alter the growth behavior of HSCs, which may lose in part their capability of dividing through alterations of the telomere-telomerase system, premature cellular senescence, and apoptosis. The opposite may also be true, and HSCs may retain after transdifferentiation a stronger regenerative capacity than CSCs. However, with HSCs, the newly formed myocytes have fetal-neonatal properties and may not reach the adult phenotype, a problem that may not affect the commitment of CSCs. Understanding the biology of HSC transdifferentiation and the molecular control of CSC growth and lineage commitment is a challenging and exciting endeavor due to the extraordinary clinical importance of myocardial regeneration for patients affected by acute MI and CHF.

Address correspondence to: Piero Anversa, Departments of Anesthesia and Medicine and Division of Cardiovascular Medicine, 75 Francis Street, Brigham and Women's Hospital, Harvard Medical School, Boston, Massachusetts, USA. Phone: 617.525.8170; Fax: 617.264.6320; E-mail: panversa@partners.org.

\footnotetext{
1. Rubart M, Field LJ. Cardiac regeneration: repopulating the heart. Annu Rev Physiol. 2006;68:29-49.

2. Bergmann O, et al. Evidence for cardiomyocyte renewal in humans. Science. 2009;324(5923):98-102.

3. Laflamme MA, Murry CE. Heart regeneration. Nature. 2011;473(7347):326-335.

4. Vunjak-Novakovic G, Lui KO, Tandon N, Chien $\mathrm{KR}$. Bioengineering heart muscle: a paradigm for regenerative medicine. Annu Rev Biomed Eng. 2011; 13:245-267.

5. Xu H, Yi BA, Chien KR. Shortcuts to making cardiomyocytes. Nat Cell Biol. 2011;13(3):191-193.

6. Kajstura J, Leri A, Finato N, Di Loreto C, Beltrami CA, Anversa P. Myocyte proliferation in end-stage cardiac failure in humans. Proc Natl Acad Sci US A. 1998;95(15):8801-8805.
}

7. Beltrami AP, et al. Evidence that human cardiac myocytes divide after myocardial infarction. NEngl JMed. 2001;344(23):1750-1757.

8. Quaini F, et al. Chimerism of the transplanted heart. NEngl J Med. 2002;346(1):5-15.

9. Urbanek $\mathrm{K}$, et al. Intense myocyte formation from cardiac stem cells in human cardiac hypertrophy. Proc Natl Acad Sci U S A. 2003;100(18):10440-10445.

10. Urbanek K, et al. Myocardial regeneration by activation of multipotent cardiac stem cells in ischemic heart failure. Proc Natl Acad Sci U S A. 2005; 102(24):8692-8697.

11. Kajstura J, et al. Cardiomyogenesis in the adult human heart. Circ Res. 2010;107(2):305-315.

12. Kajstura J, et al. Myocyte turnover in the aging human heart. Circ Res. 2010;107(11):1374-1386.
13. Leri A, Kajstura J, Anversa P. Role of cardiac stem cells in cardiac pathophysiology: a paradigm shift in human myocardial biology. Circ Res. 2011; 109(8):941-961.

14. Steinhauser ML, Lee RT. Regeneration of the heart. EMBO Mol Med. 2011;3(12):701-712.

15. Beltrami AP, et al. Adult cardiac stem cells are multipotent and support myocardial regeneration. Cell. 2003;114(6):763-776.

16. Linke A, et al. Stem cells in the dog heart are self-renewing, clonogenic, and multipotent and regenerate infarcted myocardium, improving cardiac function. Proc Natl Acad Sci U S A. 2005;102(25):8966-8971.

17. Bearzi C, et al. Human cardiac stem cells. Proc Natl Acad Sci U S A. 2007;104(35):14068-14073.

18. Limana $F$, et al. Identification of myocardial and 
vascular precursor cells in human and mouse epicardium. Circ Res. 2007;101(12):1255-1265.

19. Castaldo C, et al. CD117-positive cells in adult human heart are localized in the subepicardium, and their activation is associated with laminin-1 and alpha6 integrin expression. Stem Cells. 2008; 26(7):1723-1731.

20. Fransioli J, et al. Evolution of the c-kit-positive cell response to pathological challenge in the myocardium. Stem Cells. 2008;26(5):1315-1324.

21. Bearzi C, et al. Identification of a coronary vascular progenitor cell in the human heart. Proc Natl Acad Sci US A. 2009;106(37):15885-15890.

22. Cottage CT, et al. Cardiac progenitor cell cycling stimulated by pim-1 kinase. Circ Res. 2010; 106(5):891-901.

23. Angert D, et al. Repair of the injured adult heart involves new myocytes potentially derived from resident cardiac stem cells. Circ Res. 2011; 108(10):1226-1237.

24. Oh $\mathrm{H}$, et al. Cardiac progenitor cells from adult myocardium: homing, differentiation, and fusion after infarction. Proc Natl Acad Sci U S A. 2003; 100(21):12313-12318.

25. Smith RR, et al. Regenerative potential of cardiosphere-derived cells expanded from percutaneous endomyocardial biopsy specimens. Circulation. 2007;115(7):896-908

26. Matsuura K, et al. Transplantation of cardiac progenitor cells ameliorates cardiac dysfunction after myocardial infarction in mice. J Clin Invest. 2009;119(8):2204-2217.

27. $\mathrm{Bu} \mathrm{L}$, et al. Human ISL1 heart progenitors generate diverse multipotent cardiovascular cell lineages. Nature. 2009;460(7251):113-117.

28. Oikonomopoulos A, et al. Wnt signaling exerts an antiproliferative effect on adult cardiac progenitor cells through IGFBP3. Circ Res. 2011; 109(12):1363-1374

29. Chong JJ, et al. Adult cardiac-resident MSC-like stem cells with a proepicardial origin. Cell Stem Cell. 2011;9(6):527-540.

30. Urbanek K, et al. Stem cell niches in the adult mouse heart. Proc Natl Acad Sci U S A. 2006 103(24):9226-9231.

31. Anversa P, Leri A, Kajstura J. Cardiac regeneration. J Am Coll Cardiol. 2006;47(9):1769-1776.

32. Engel FB, et al. p38 MAP kinase inhibition enables proliferation of adult mammalian cardiomyocytes. Genes Dev. 2005;19(10):1175-1187.

33. Kühn B, et al. Periostin induces proliferation of differentiated cardiomyocytes and promotes cardiac repair. Nat Med. 2007;13(8):962-969.

34. Bersell K, Arab S, Haring B, Kühn B. Neuregulin1/ErbB4 signaling induces cardiomyocyte proliferation and repair of heart injury. Cell. 2009; 138(2):257-270.

35. Kubin T, et al. Oncostatin M is a major mediator of cardiomyocyte dedifferentiation and remodeling. Cell Stem Cell. 2011;9(5):420-432.

36. Hsieh PC, et al. Evidence from a genetic fate-mapping study that stem cells refresh adult mammalian cardiomyocytes after injury. Nat Med. 2007; 13(8):970-974.

37. Murry CE, Lee RT. Development biology. Turnover after the fallout. Science. 2009;324(5923):47-48.

38. Parmacek MS, Epstein JA. Cardiomyocyte renewal. NEngl J Med. 2009;361(1):86-88.

39. Olivetti $G$, et al. Apoptosis in the failing human heart. N Engl J Med. 1997;336(16):1131-1141.

40. Mahajan VS, Jarolim P. How to interpret elevated cardiac troponin levels. Circulation. 2011; 124(21):2350-2354.

41. Omland T, et al. Prevention of Events with Angi otensin Converting Enzyme Inhibition (PEACE) Trial Investigators. A sensitive cardiac troponin T assay in stable coronary artery disease. $N$ Engl J Med. 2009;361(26):2538-2547.
42. Hosoda T, et al. Clonality of mouse and human cardiomyogenesis in vivo. Proc Natl Acad Sci U S A. 2009;106(40):17169-17174.

43. Fuchs E, Horsley V. Ferreting out stem cells from their niches. Nat Cell Biol. 2011;13(5):513-518.

44. Lo Celso C, Scadden DT. The haematopoietic stem cell niche at a glance. J Cell Sci. 2011; 124(pt 21):3529-3535.

45. Gonzalez A, et al. Activation of cardiac progenitor cells reverses the failing heart senescent phenotype and prolongs lifespan. Circ Res. 2008;102(5):597-606.

46. Kim SK, Miller JH. Natural history and distribution of bone and bone marrow infarction in sickle hemoglobinopathies. J Nucl Med. 2002;43(7):896-900.

47. Francque S, et al. Multifactorial aetiology of hepatic infarction: a case report with literature review. EurJ Gastroenterol Hepatol. 2004;16(4):411-415.

48. Berland T, Oldenburg WA. Acute mesenteric ischemia. Curr Gastroenterol Rep. 2008;10(3):341-346

49. Maude GH. Bone marrow infarction in sickle cell anemia. Blood. 1984;63(1):243.

50. Leong FT, Freeman LJ. Acute renal infarction. JR Soc Med. 2005;98(3):121-122.

51. Lopez LR, Schocket AL, Stanford RE, Claman HN, Kohler PF. Gastrointestinal involvement in leukocytoclastic vasculitis and polyarteritis nodosa. J Rheumatol. 1980;7(5):677-684.

52. Cliffe LJ, Humphreys NE, Lane TE, Potten CS, Booth C, Grencis RK. Accelerated intestinal epithelial cell turnover: a new mechanism of parasite expulsion. Science. 2005;308(5727):1463-1465.

53. Holden C. Scientists find heart stem cells. ScienceNow. Web site. http://news.sciencemag.org/sciencenow/ 2009/07/02-01.html. Accessed December 10, 2012.

54. Hsu YC, Fuchs E. A family business: stem cell progeny join the niche to regulate homeostasis. Nat Rev Mol Cell Biol. 2012;13(2):103-114.

55. Leri A. Human cardiac stem cells: the heart of a truth. Circulation. 2009;120(25):2515-2518

56. Verfaillie CM. "Adult" stem cells: tissue specific or not? In: Lanza R, eds. Handbook of Stem Cells. New York, New York, USA: Elsevier Academic Press; 2004:13-20.

57. Kretzschmar K, Watt FM. Lineage tracing. Cell. 2012;148(1-2):33-45.

58. Suh H, Consiglio A, Ray J, Sawai T, D’Amour KA, Gage $\mathrm{FH}$. In vivo fate analysis reveals the multipotent and self-renewal capacities of Sox $2+$ neural stem cells in the adult hippocampus. Cell Stem Cell. 2007;1(5):515-528

59. Anversa P, Kajstura J, Leri A, Loscalzo J. Tissue-specific adult stem cells in the human lung. Nat Med. 2011;17(9):1038-1039.

60. Torella D, et al. Cardiac stem cell and myocyte aging, heart failure, and insulin-like growth factor-1 overexpression. Circ Res. 2004;94(4):514-524.

61. Ferreira-Martins J, et al. Cardiomyogenesis in the developing heart is regulated by c-kit-positive cardiac stem cells. Circ Res. 2012;110(5):701-715

62. Ferreira-Martins J, et al. Spontaneous calcium oscillations regulate human cardiac progenitor cell growth. Circ Res. 2009;105(8):764-774.

63. Dimmeler S, Zeiher AM, Schneider MD. Unchain my heart: the scientific foundations of cardiac repair. J Clin Invest. 2005;115(3):572-583.

64. Fazel S, et al. Cardioprotective c-kit+ cells are from the bone marrow and regulate the myocardial balance of angiogenic cytokines. J Clin Invest. 2006; 116(7):1865-1877.

65. Slack JM, Tosh D. Transdifferentiation and metaplasia--switching cell types. Curr Opin Genet Dev. 2001;11(5):581-586.

66. Leri A, Kajstura J, Anversa P. Cardiac stem cells and mechanisms of myocardial regeneration. Physiol Rev. 2005;85(4):1373-1416

67. Blau HM, et al. Plasticity of the differentiated state. Science. 1985;230(4727):758-766.

68. Mezey E. Commentary: on bone marrow stem cells and openmindedness. Stem Cells Dev. 2004; 13(1):147-152.

69. Pomerantz J, Blau HM. Nuclear reprogramming: a key to stem cell function in regenerative medicine. Nat Cell Biol. 2004;6(9):810-816.

70. Harris RG, Herzog EL, Bruscia EM, Grove JE, Van Arnam JS, Krause DS. Lack of a fusion requirement for development of bone marrow-derived epithelia. Science. 2004;305(5680):90-93.

71 . Orlic D, et al. Bone marrow cells regenerate infarcted myocardium. Nature. 2001;410(6829):701-705.

72. Orlic D, et al. Mobilized bone marrow cells repair the infarcted heart, improving function and survival. Proc Natl Acad Sci U S A. 2001;98(18):10344-10349.

73. Yoon YS, et al. Clonally expanded novel multipotent stem cells from human bone marrow regenerate myocardium after myocardial infarction. J Clin Invest. 2005;115(2):326-338

74. Murasawa S, Kawamoto A, Horii M, Nakamori $\mathrm{S}$, Asahara T. Niche-dependent translineage commitment of endothelial progenitor cells, not cell fusion in general, into myocardial lineage cells. Arterioscler Thromb Vasc Biol. 2005;25(7):1388-1394.

75. Rota $\mathrm{M}$, et al. Bone marrow cells adopt the cardiomyogenic fate in vivo. Proc Natl Acad Sci U S A. 2007;104(45):17783-17788

76. Haider HKh, Jiang S, Idris NM, Ashraf M. IGF-1-overexpressing mesenchymal stem cells accelerate bone marrow stem cell mobilization via paracrine activation of SDF-1alpha/CXCR4 signaling to promote myocardial repair. Circ Res. 2008;103(11):1300-1308.

77. Hatzistergos KE, et al. Bone marrow mesenchymal stem cells stimulate cardiac stem cell proliferation and differentiation. Circ Res. 2010;107(7):913-922.

78. Suzuki G, Iyer V, Lee TC, Canty JM Jr. Autologous mesenchymal stem cells mobilize cKit + and CD133+ bone marrow progenitor cells and improve regional function in hibernating myocardium. Circ Res. 2011;109(9):1044-1054.

79. Murry CE, et al. Haematopoietic stem cells do not transdifferentiate into cardiac myocytes in myocardial infarcts. Nature. 2004;428(6983):664-668.

80. Balsam LB, Wagers AJ, Christensen JL, Kofidis T, Weissman IL, Robbins RC. Haematopoietic stem cells adopt mature haematopoietic fates in ischaemic myocardium. Nature. 2004;428(6983):668-673.

81. Chien KR. Stem cells: lost in translation. Nature. 2004;428(6983):607-608.

82. Loffredo FS, Steinhauser ML, Gannon J, Lee RT. Bone marrow-derived cell therapy stimulates endogenous cardiomyocyte progenitors and promotes cardiac repair. Cell Stem Cell. 2011;8(4):389-398.

83. Abdel-Latif A, et al. Adult bone marrow-derived cells for cardiac repair: a systematic review and meta-analysis. Arch Intern Med. 2007;167(10):989-997.

84. Kang S, Yang YJ, Li CJ, Gao RL. Effects of intracoronary autologous bone marrow cells on left ventricular function in acute myocardial infarction: a systematic review and meta-analysis for randomized controlled trials. Coron Artery Dis. 2008;19(5):327-335.

85 . Kajstura J, et al. Bone marrow cells differentiate in cardiac cell lineages after infarction independently of cell fusion. Circ Res. 2005;96(1):127-137.

86. Nakamura T, Schneider MD. The way to a human's heart is through the stomach: visceral endodermlike cells drive human embryonic stem cells to a cardiac fate. Circulation. 2003;107(21):2638-2639.

87. Dispersyn GD, et al. Dissociation of cardiomyocyte apoptosis and dedifferentiation in infarct border zones. Eur Heart J. 2002;23(11):849-857.

88. Oka T, et al. Genetic manipulation of periostin expression reveals a role in cardiac hypertrophy and ventricular remodeling. Circ Res. 2007; 101(3):313-321.

89. Anversa P, Vitali-Mazza L, Loud AV. Morphometric and autoradiographic study of developing ventricular and atrial myocardium in fetal rats. Lab Invest. 1975;33(6):696-705. 
90. Anversa P, Olivetti G, Bracchi PG, Loud AV. Postnatal development of the $\mathrm{M}$-band in rat cardiac myofibrils. Circ Res. 1981;48(4):561-568.

91. Morrisey EE. Rewind to recover: dedifferentiation after cardiac injury. Cell Stem Cell. 2011;9(5):387-388.

92. Lunde $\mathrm{K}$, et al. Intracoronary injection of mononuclear bone marrow cells in acute myocardial infarction. NEngl J Med. 2006;355(12):1199-1209.

93. Meyer GP, et al. Intracoronary bone marrow cell transfer after myocardial infarction: eighteen months' follow-up data from the randomized, controlled BOOST (BOne marrOw transfer to enhance ST-elevation infarct regeneration) trial. Circulation. 2006;113(10):1287-1294.

94. Beitnes JO, et al. Long-term results after intracoronary injection of autologous mononuclear bone marrow cells in acute myocardial infarction: the ASTAMI randomised, controlled study. Heart. 2009;95(24):1983-1989.

95. Traverse JH, et al. Cardiovascular Cell Therapy Research Network. Effect of intracoronary delivery of autologous bone marrow mononuclear cells 2 to
3 weeks following acute myocardial infarction on left ventricular function: the LateTIME randomized trial. JAMA. 2011;306(19):2110-2119.

96. Perin EC, et al. Effect of transendocardial delivery of autologous bone marrow mononuclear cells on functional capacity, left ventricular function, and perfusion in chronic heart failure: the FOCUSCCTRN trial. JAMA. 2012;307(16):1717-1726.

97. Bolli $\mathrm{R}$, et al. Cardiac stem cells in patients with ischaemic cardiomyopathy (SCIPIO): initial results of a randomised phase 1 trial. Lancet. 2011; 378(9806):1847-1857.

98. D'Amario D, et al. Functionally competent cardiac stem cells can be isolated from endomyocardial biopsies of patients with advanced cardiomyopathies. Circ Res. 2011;108(7):857-861.

99. Chimenti C, et al. Senescence and death of primitive cells and myocytes lead to premature cardiac aging and heart failure. Circ Res. 2003;93(7):604-613.

100.Dimmeler S, Leri A. Aging and disease as modifiers of efficacy of cell therapy. Circ Res. 2008; 102(11):1319-1330.
101. D'Amario D, et al. Insulin-like growth factor-1 receptor identifies a pool of human cardiac stem cells with superior therapeutic potential for myocardial regeneration. Circ Res. 2011;108(12):1467-1481.

102.Cesselli D, et al. Effects of age and heart failure on human cardiac stem cell function. Am J Pathol. 2011;179(1):349-366.

103. Rota M, et al. Diabetes promotes cardiac stem cell aging and heart failure, which are prevented by deletion of the p66shc gene. Circ Res. 2006;99(1):42-52.

104.Yang S, et al. Activated IGF-1R inhibits hyperglycemia-induced DNA damage and promotes DNA repair by homologous recombination. Am J Physiol Renal Physiol. 2005;289(5):F1144-F1152.

105. Ratajczak MZ, et al. CD34+, kit+, rhodamine123(low) phenotype identifies a marrow cell population highly enriched for human hematopoietic stem cells. Leukemia. 1998;12(6):942-950.

106.Ziebart T, et al. Sustained persistence of transplanted proangiogenic cells contributes to neovascularization and cardiac function after ischemia. Circ Res. 2008;103(11):1327-1334. 